ORIGINAL ARTICLE

\title{
Supermarket baker's asthma: how accurate is routine health surveillance?
}

\author{
A Brant, S Nightingale, J Berriman, C Sharp, J Welch, A J Newman Taylor, P Cullinan
}

Occup Environ Med 2005;62:395-399. doi: 10.1136/oem.2004.014639

See end of article for authors' affiliations .....................

Correspondence to: Dr A Brant, Department of Occupational and Environmental Medicine, National Heart and Lung Institute, Imperial College, Ib Manresa Rd, London SW3 6LR, UK; a.brant@ ic.ac.uk

Accepted 1 December 2004

\begin{abstract}
Background: Regular health surveillance is commonly recommended for workers exposed to occupational antigens but little is known about how effective it is in identifying cases.

Aims: To report one large company's surveillance and compare its findings with those of a standard crosssectional survey in the same workforce.

Methods: A supermarket company with 324 in-store bakeries producing bread from raw ingredients conducted a three-stage health surveillance programme in around 3000 bakery employees. The first stage involved the administration of a simple respiratory questionnaire. If chest symptoms were present a second questionnaire focusing on their work relationship was administered. If positive a blood sample was requested for the measurement of specific lgE to flour and fungal $\alpha$-amylase. The results were compared to an independent cross-sectional survey of employees in 20 of the company's stores.

Results: Two hundred and ninety nine (92\%) of the company's bakeries took part in surveillance. The overall employee response for the first stage was 77\%; a quarter of those with respiratory symptoms reported that they were work related. Seventy four $(61 \%)$ of those with work related chest symptoms had a measurement of specific lgE to either flour or fungal $\alpha$-amylase, of whom $30(41 \%)$ had a positive result. Surveillance estimated that $1 \%$ of bakery employees ( $1 \%$ bakers, $2 \%$ managers, $0.6 \%$ confectioners) had work related symptoms with specific lgE. This compared with $4 \% 17.5 \%$ bakers, $3.3 \%$ managers, $0 \%$ confectioners) in the cross-sectional survey ( $n=166,93 \%$ response).

Conclusion: Comparison with a standard cross-sectional survey suggests that routine surveillance can underestimate the workplace burden of disease. The reasons may include technical or resource issues and uncertainties over confidentiality or the perceived consequences of participation. More research needs to be done looking into the design and efficacy of surveillance in occupational asthma.
\end{abstract}

$\mathrm{R}$ outine health surveillance is commonly practised-and generally enforced by regulation-in workplaces where there is exposure to known respiratory sensitisers and a residual risk of occupational asthma. Its aims include: the identification of individuals who have developed occupational asthma at an early and reversible stage of their disease to allow, as a method of tertiary prevention, their removal from further exposure; ${ }^{12}$ and the identification of a need to improve exposure control more widely. Surveillance may also be used to provide an estimate of the prevalence of disease in a workplace.

Previous studies ${ }^{3}{ }^{4}$ have suggested that routine surveillance can be successful in reducing the frequency of disease. Regulation in Ontario mandated employers to monitor the exposure levels of diisocyanates in the workplace and implement a surveillance programme including six monthly respiratory questionnaires and annual spirometry. ${ }^{4}$ Initially the number of identified cases increased, but subsequently there was a reduction in reports of diisocyanate induced occupational asthma, although not of cases of occupational asthma not subject to control and surveillance. Those with the disease were identified earlier and with less severe disease. As surveillance was part of an overall prevention strategy it is difficult to know the extent of its contribution to these changes. In the platinum industry, individuals identified at surveillance by a positive specific skin prick test to platinum salts were prevented from developing chronic asthma symptoms by removing them from exposure. ${ }^{3}$ Within the same industry, it has been suggested that earlier identification of sensitised workers through regular surveillance, and their earlier removal from exposure, was followed by an improved prognosis. ${ }^{5}$
Health surveillance is predicated on it being an effective method of identifying cases but evidence that this is so, using current methods, is limited. Gordon et al for example, demonstrated that bakery workers who reported respiratory symptoms in a screening questionnaire were no more likely to have asthma (or occupational asthma) than those who responded negatively. ${ }^{6}$ We decided to explore this apparent discrepancy in a population of workers employed in supermarket bakeries, all producing bread from raw ingredients. Baker's asthma arising from exposure to flour and enzymes (which improve the baking process) remains a commonly reported cause of occupational asthma. ${ }^{7}$ We wanted to estimate how accurate routine workplace health surveillance is at estimating the prevalence of occupational asthma. We did this by comparing the results of a company's surveillance programme with the results of an independent crosssectional survey in the same workforce, a well established epidemiological method or standard for estimating the prevalence of disease in the workplace. We used work related chest symptoms with serum specific IgE to either flour or fungal $\alpha$-amylase as a workable definition of baker's asthma; the combination is an acceptable surrogate for occupational asthma. ${ }^{8}$

\section{METHODS}

\section{Company surveillance}

A large supermarket company had 324 stores with in-house bakeries throughout the UK and undertakes its own annual health surveillance. We reviewed the results from 2001-02. Employees were categorised by their primary job role: "bakers" mix raw ingredients (including wheat flour and enzymes) and make bread; "confectioners" finish and 
decorate pastries; "managers" perform several tasks and are themselves often bakers. Other bakery workers, including packing and counter staff, were not included in the surveillance as they were not thought to be at risk of developing occupational asthma. The surveillance was undertaken by the company's occupational health advisors (OHA), registered nurses with higher qualifications in occupational health. All had received training into baker's asthma.

Surveillance was undertaken in three stages:

- Stage 1: a short questionnaire was administered by an occupational health advisor to identify employees with respiratory symptoms, including a recurrent blocked or runny nose, recurring sore or runny eyes, coughing, and chest tightness.

- Stage 2: if any respiratory symptoms, upper or lower, were reported a further questionnaire focusing on their work relatedness was administered by the occupational health advisor.

- Stage 3: if work related chest symptoms were reported the employee was requested to arrange for a blood sample to be collected through his or her general practice and sent to an independent laboratory at the Department of Occupational and Environmental Medicine, Royal Brompton Hospital. Serum IgE to wheat flour (a mix of five Canadian and English wheat flours) and fungal $\alpha$ amylase (Novo Nordisk) were measured by radioallergosorbent (RAST) assay. The result was positive if RAST binding was $\geqslant 2 \%$. Blood results were routinely forwarded to the occupational health department.

As a readily available means of estimating dust exposure, information was collected on the size and annual sales of each bakery.

\section{Cross-sectional survey}

We undertook an independent cross-sectional survey of all bakery employees in 20 scratch bakeries from the same company and over the same time period as the surveillance. For logistical reasons we selected stores from a limited geographical area; 25 stores with a scratch bakery fell within this area and 20 were randomly selected for the survey. These bakeries did not differ in size, number of bakery employees or baking processes from bakeries in the company's stores in the rest of the country. Multiple visits by the investigator (AB) were required to optimise response rates.

Each employee was administered a questionnaire enquiring into their employment history and "any respiratory symptoms" over the last year: chest tightness, difficulty breathing, chest wheezing or whistling, running or itchiness of the nose or eyes, or sneezing. The symptoms were considered "work related" if they reported improvement after two or more days away from work. As above, and in the same laboratory, serum IgE to wheat flour and fungal $\alpha$ amylase were measured by RAST assay and considered positive if binding was $\geqslant 2 \%$. We report here, as for surveillance, the results for "bakers", "managers", and "confectioners" as above.

\section{Analysis}

Comparisons between proportions were performed by $\chi^{2}$ analysis. We used a normal approximation to estimate confidence limits for each proportion; except in the case of bakery managers, who were few, and for whom we used an exact method.

Approval for the survey was provided by the ethics committee of Royal Brompton and Harefield Hospital NHS Trust. Further clinical evaluation was offered to subjects if they so wished.

\section{RESULTS}

\section{Supermarket bakery health surveillance (fig 1)}

Two hundred and ninety nine (92\%) of the company's stores provided results that were suitable for analysis. These stores employed 2975 bakers, managers, and confectioners. The overall employee response rates were $77 \%(n=2279)$ for stage $1,96 \%(n=469)$ for stage 2 , and $61 \%(n=74)$ for stage 3. Response rates were higher for bakers than other employees at stage 1 , but were lower at stage 3 with proportionally fewer bakers than managers or confectioners having a blood test.

Twenty two per cent of those who underwent surveillance reported "any" respiratory symptoms. A quarter $(26 \%)$ of these reported work related chest symptoms, a proportion that was higher in bakers and managers than confectioners. Thirty $(41 \%)$ of the 74 employees who provided a blood sample had specific IgE to at least one bakery allergen. A higher proportion of managers than bakers and confectioners had a positive blood test, but the numbers were low. Twenty eight of the 30 positive samples had specific IgE to flour, 13 had specific IgE to both flour and fungal $\alpha$-amylase, and two to fungal $\alpha$-amylase alone.

The median annual sales figure for stores with one or more employees reporting work related chest symptoms was higher than for stores without such employees $(p=0.049)$. The difference was similar for stores with employees with work related chest symptoms and specific IgE, but did not reach significance $(p=0.088)$. The size of the store was not associated with either respiratory symptoms or specific IgE.

\section{Cross-sectional survey (table 1)}

One hundred and fifty four of the total population of 166 bakers, managers, and confectioners from the 20 stores took part in the survey. The response rate was high in each group: bakers (89\%), managers (100\%), and confectioners (95\%). All answered the questionnaire, and $139(90 \%)$ provided a blood sample. The main reason for non-response was unavailability due to work shifts.

Respiratory symptoms of any type were reported commonly by workers in each job group. Work related chest symptoms were reported by $16 \%$ of employees, most frequently by bakers. Twenty three (17\%) employees had IgE antibodies to either flour or fungal $\alpha$-amylase. Seven $(5 \%)$ with specific IgE to one or both allergens, six bakers and one confectioner, reported work related chest symptoms.

\section{Comparison of surveillance and survey}

The prevalence of employees who reported "any respiratory symptoms" was higher at survey than surveillance (64\% v $22 \%)$; however, the proportions of these who reported that their symptoms were work related were each $26 \%$. Of those with work related chest symptoms who subsequently underwent venesection, $32 \%$ at survey and $41 \%$ at surveillance had specific IgE to a bakery allergen $(p=0.46)$.

Table 2 outlines a comparison of the numbers of employees with both work related chest symptoms and specific sensitisation identified at survey or at surveillance. These numbers have been related to the total eligible, rather than respondent, populations. Overall, the survey estimated a prevalence of work related symptoms with specific sensitisation four times that estimated by surveillance; among bakers this figure was 7.5 -fold. Both differences were statistically significant.

\section{DISCUSSION}

As far as we are aware this is the first systematic evaluation of the validity of a surveillance programme for occupational asthma. Using, as a workable case definition, the combination of work related chest symptoms and specific 
Total

(Eligible for Stage 1)

Stage 1

Stage 2

Stage 3

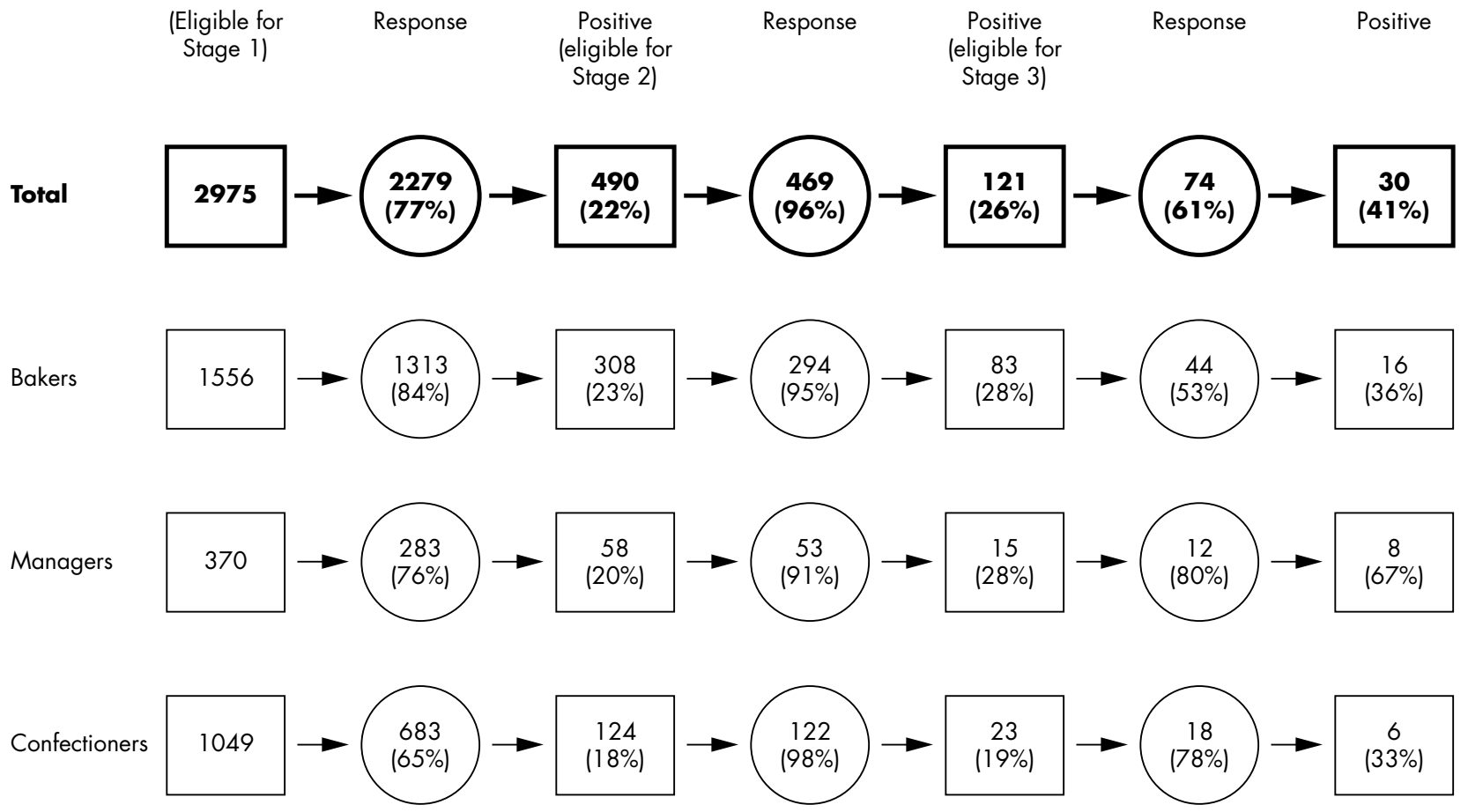

Figure 1 Supermarket bakery surveillance.

immunology, the estimate of disease prevalence from a crosssectional survey of a sample of supermarket bakery workers from a single company was four times greater than the company's own health surveillance; the difference for bakers was over sevenfold. The findings could have important implications for other occupational asthma surveillance programmes.

This supermarket company faced several challenges in achieving full coverage and completion of their surveillance. These included the large size and broad dispersal of the workforce (around 3000 bakers, managers, and confectioners at over 300 sites), their variable work shifts and work patterns, and a relatively high rate of employee turnover. Alongside this surveillance programme each of the firm's OHAs had responsibility for around 7000 employees and up to 40 different stores often at widespread locations. Variations in individual practice were inevitable but very difficult to quantify. Nevertheless, surveillance was sufficiently sensitive to detect a crude exposure-response relation; bakers, most likely to have highest exposure to dust, were more likely to have work related chest symptoms with specific sensitisation than workers in other jobs. Furthermore, workers with work related chest symptoms were more likely to be employed in stores with higher yearly sales, probably correlated with bread production and consequently dust exposure.

The differences in outcomes between the two methods may in part reflect the differences in response rates. The company's surveillance was undertaken in a step-wise manner, an approach which is used elsewhere in the baking industry. ${ }^{9}$ Most of the company's stores were covered but individual response rates $(77 \%, 96 \%$, and $61 \%$ at stages 1,2 , and 3, respectively) indicate that rates of initial participation and blood collection were relatively low. At stage 1, bakers (at highest risk of disease) were the most likely to participate; by stage 3 the opposite was true. In contrast, the design of the epidemiological survey avoided a staged procedure and allowed a more concentrated examination of the issue. Almost all eligible employees, in all job groups, took part. Non-responders may differ importantly from those who participate; they may be more likely to have disease and less

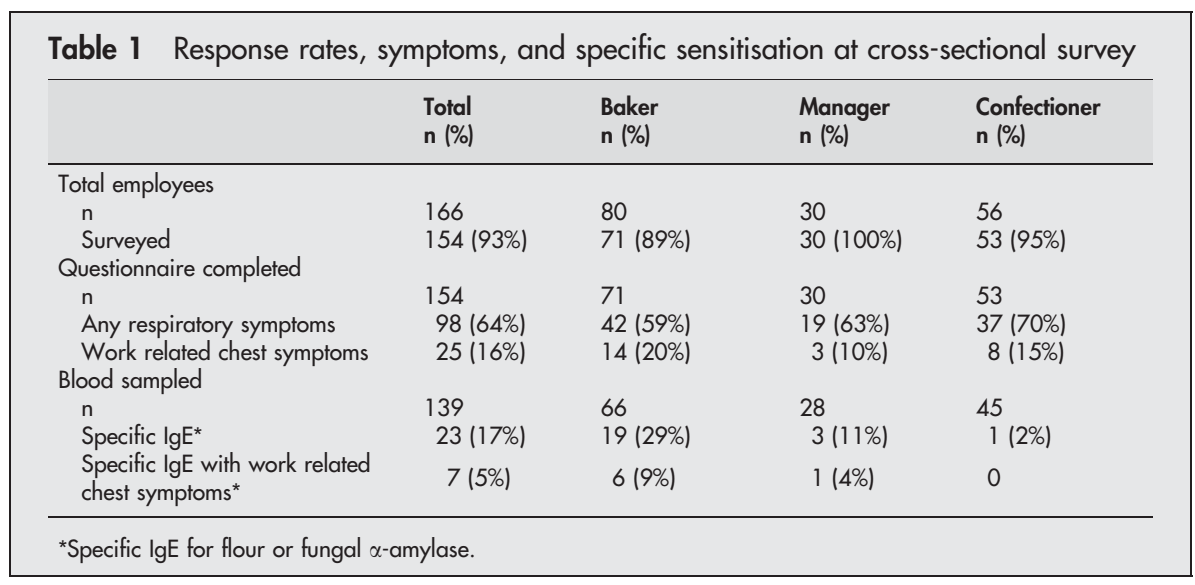




\begin{tabular}{|c|c|c|c|c|}
\hline & $\begin{array}{l}\text { Total } \\
\text { n (\%; } \pm 2 \text { SD) }\end{array}$ & $\begin{array}{l}\text { Baker } \\
\text { n (\%; } \pm 2 \text { SD) }\end{array}$ & $\begin{array}{l}\text { Manager } \\
\mathrm{n}(\% ; \pm 2 \text { SD) }\end{array}$ & $\begin{array}{l}\text { Confectioner } \\
\text { n (\%; } \pm 2 \text { SD) }\end{array}$ \\
\hline \multirow{2}{*}{$\begin{array}{l}\text { Surveillance } \\
\text { Work related chest } \\
\text { symptoms with specific lgE* }\end{array}$} & 2975 & 1556 & 370 & 1049 \\
\hline & $30(1 \% ; 0.6-1.4)$ & $16(1 \% ; 0.5-1.5)$ & $8(2 \% ; 0.5-3.5)$ & $6(0.6 \% ; 0.1-1.1)$ \\
\hline \multirow{2}{*}{$\begin{array}{l}\text { Survey } \\
\text { Work related chest } \\
\text { symptoms with specific } \lg \mathrm{E}^{*}\end{array}$} & 166 & 80 & 30 & 56 \\
\hline & $7(4 \% ; 1-7)$ & $6(7.5 \% ; 1.6-13.4)$ & $1(3.3 \% ; 0.1-17.2)$ & 0 \\
\hline
\end{tabular}

willing to be identified, particularly if they perceive adverse consequences from this. Subsequent involvement of the GP in obtaining blood samples (as opposed to sampling at the time of assessment, as at survey) may also have reduced participation in the final stage of surveillance.

The clearest difference between the two methods was in the reporting of "any" respiratory symptoms, by three times as many employees at survey than at surveillance. Of the 115 employees who participated in both survey and surveillance, only one third of those reporting "any" respiratory symptoms in the survey also reported them in the surveillance. The difference may reflect different thresholds of symptom identification. At surveillance, any work relation of chest symptoms was identified not at stage 1, but at stage 2 . Too general a screening questionnaire, rather than being over sensitive as is often the criticism, may inadvertently provide an opportunity for symptoms to be overlooked. If employees had at surveillance reported a similar proportion of "any" respiratory symptoms to those at survey $(64 \%)$, the estimate of sensitisation with work related chest symptoms at surveillance would be about 3\%, a result similar to that from the cross-sectional survey.

There are no validated questionnaires for the detection of occupational asthma. In our cross-sectional survey the work related questions were the same as those used in a study of occupational asthma in the detergent industry where we estimated their positive predictive value, in combination with positive serology, to be about $75 \%{ }^{8}$ In the current study the wording of the surveillance questionnaire differed only slightly due to the requirements of the company's programme. These differences are unlikely to explain more than a small part of the reporting differences. Similar proportions of respiratory symptoms reported at survey and surveillance were reported to be work related. Furthermore, when work related chest symptoms were present (and blood taken), both methods found a similar proportion of employees with bakery allergen specific IgE. These consistencies indicate some internal validity in the comparison of the results of survey and surveillance.

Apparent under-reporting at surveillance may also have arisen from employees' reluctance to divulge symptoms, perhaps through concerns or uncertainty over the consequences of doing so. The cross-sectional survey was conducted by a research group external to the company and assured complete confidentiality; this may have been less easy to ensure at surveillance. Despite the best efforts of an employer, some individuals may continue to respond inappropriately to surveillance. Education and information for the workforce, and protection of employment conditions for those with occupational illness, may address some of these issues but are dependent on an employer's time and resources.

Cross-sectional surveys, such as ours here, are a well established method of estimating the prevalence of disease in the workplace and the frequencies of any or work related chest respiratory symptoms in this survey were similar to other epidemiological investigations in bakery workforces. ${ }^{10} 11$
Nevertheless we considered whether the differences we found might reflect an unrepresentatively high measure of disease at the cross-sectional survey. This might have occurred, for example, if the 20 surveyed stores, selected for convenience, were not representative of all the company's UK stores. The stores however were no different in size, employee numbers, age, or baking process to the rest of those owned by the company. Except for the cases of seven employees (from two stores), all the surveys were undertaken after the surveillance programme; conceivably, experience with surveillance may have directed responses to the survey questionnaires but we cannot know in which direction.

Audits of occupational health surveillance schemes have highlighted the need for quality assurance. ${ }^{12}{ }^{13}$ Little, though, is known about the appropriate design of a surveillance scheme in occupational asthma. In part this may be because of the difficulties with the accurate diagnosis of disease, made clinically from a combination of history, lung function, airway responsiveness, specific immunology, and inhalation testing. Alone, except possibly inhalation testing, none of these is sufficient for a diagnosis. Questionnaires are sensitive and easy to administer but are not specific, single measurements of lung function are neither specific nor sensitive, immunology is not specific and limited to a few allergens, and inhalation testing is available only in a few specialist centres. Used in combination, diagnostic accuracy improves, and the design of a surveillance scheme will depend on how far along a diagnostic pathway it wishes to proceed. It will also depend on the purpose of the surveillance; health surveillance is not the same as diagnosis. The nature of the allergen, employer resources, local occupational health services, and access to diagnostic expertise will all impact on how best a surveillance scheme should be designed.

Our experience suggests that routine surveillance can importantly underestimate the workplace burden of occupational asthma. This leads to a failure to identify cases at an early and potentially remediable stage of disease and to the loss of an opportunity to recognise a need to improve exposure control. The reasons may include technical or resource issues and uncertainties over confidentiality or the perceived consequences of participation. More research needs to be done looking into the design and efficacy of surveillance in occupational asthma.

\section{Authors' affiliations}

A Brant, S Nightingale, J Berriman, C Sharp, J Welch, A J Newman Taylor, P Cullinan, Department of Occupational and Environmental Medicine, National Heart and Lung Institute, Imperial College, London, UK

Competing interests: none declared

\section{REFERENCES}

1 Chan-Yeung M, MacLean L, Paggiaro PL. Follow-up study of 232 patients with occupational asthma caused by western red cedar (Thuja plicata). J Allergy Clin Immunol 1987;79:792-6. 
2 Mapp CE, Corona PC, De Marzo N, et al. Persistent asthma due to isocyanates. A follow-up study of subjects with occupational asthma due to toluene diisocyanate (TDI). Am Rev Respir Dis 1988;137:1326-9.

3 Merget R, Caspari C, Dierkes-Globisch A, et al. Effectiveness of a medical surveillance program for the prevention of occupational asthma caused by platinum salts: a nested case-control study. J Allergy Clin Immunol 2001;107:707-12.

4 Tarlo SM, Liss GM, Yeung KS. Changes in rates and severity of compensation claims for asthma due to diisocyanates: a possible effect of medical surveillance measures. Occup Environ Med 2002;59:58-62.

5 Brooks SM, Baker DB, Gann PH, et al. Cold air challenge and platinum skin reactivity in platinum refinery workers. Bronchial reactivity precedes skin prick response. Chest 1990;97:1401-7.

6 Gordon SB, Curran AD, Murphy j, et al. Screening questionnaires for bakers' asthma-are they worth the effort? Occup Med (Lond) 1997;47:361-6.
7 McDonald JC, Keynes HL, Meredith SK. Reported incidence of occupational asthma in the United Kingdom, 1989-97. Occup Environ Med 2000:57:823-9.

8 Cullinan P, Harris JM, Newman Taylor AJ, et al. An outbreak of asthma in a modern detergent factory. Lancet 2000;356:1899-900.

9 Smith TA. Preventing baker's asthma: an alternative strategy. Occup Med (Lond) 2004;54:21-7.

10 Jeffrey P, Griffin P, Gibson M, et al. Small bakeries-a cross-sectional study of respiratory symptoms, sensitization and dust exposure. Occup Med (Lond) 1999;49:237-41.

11 Cullinan P, Lowson D, Nieuwenhuijsen MJ, et al. Work related symptoms, sensitisation, and estimated exposure in workers not previously exposed to flour. Occup Environ Med 1994;51:579-83.

12 Smedley J, Coggon D. Health surveillance for hospital employees exposed to respiratory sensitizers. Occup Med (Lond) 1996;46:33-6.

13 Murphy E, Harrison J, Beach J. Implementation of statutory occupational respiratory health surveillance. Occup Med (Lond) 2002;52:497-502. 